\title{
The Fine Structure of the Adrenal Gland of the Indian Sheath- Tailed Bat, Taphozous longimanus (Hardwicke)
}

\author{
${ }^{1}$ A. A. Nerkar, ${ }^{2}$ M. M. Gadegone \\ ${ }^{1,2}$ Cell Biology Section, Department of Zoology, Institute of Science, R. T. Road, Civil Lines, Nagpur 440001, \\ India
}

\begin{abstract}
The Indian sheath-tailed bat, Taphozous longimanus at Nagpur (Maharashtra State) shows continuous asynchronous breeding, exhibiting aseasonal polyoestry. The adrenal gland of Taphozous is made up of the steroidogenic and chromaffin cells. The ultrastructural features of the glomerulosa cells during estrus are inconspicuous Golgi complex mitochondria with vesicular cristae, a few lipid droplets and smooth endoplasmic reticulum with vesicular cisternae. Numerous mitochondria, smooth endoplasmic reticulum in the form of interconnected vesicles scattered throughout the cytoplasm and large numbers of lipid droplets are the characteristics of the cells of zona fasciculata. Adrenal gland of pregnant female shows striking differences in ultrastrctural features than those observed in the adrenal gland of non-pregnant female. During pregnancy, the cells of zona glomerulosa are hypertrophied and synthetically very active than that observed during estrus. The cells of zona fasciculata show mitochondria with tubular and lamellar cristae. There is an increase in GolgiSER area during pregnancy as compare to estrus. Lipid droplets are more than those observed in the cells of zona glomerulosa and zona fasciculata of estrus adrenal. The close association of lipid droplets with smooth endoplasmic reticulum and mitochondria suggests the active biosynthesis of steroid hormones. Adrenal medulla consists of two types of cells depending on the presence of secretory granules. Nor-epinephrine cells are predominant in the medulla of pregnant bat while epinephrine cells are predominant in the medulla of estrus bat.
\end{abstract}

Keywords- bat, adrenal cortex, medulla, ultrastructure, reproductive cycle.

\section{Introduction}

Successful reproduction in vertebrates depends on the endocrine system, which must have the capacity to function within and adapt to a changing physical- and social environment. Although there are many different neuroendocrine components involved in regulating reproduction and the response to changing environments, the hypothalamic-pituitary-adrenal (HPA) axis and the hypothalamic-pituitary-gonadal (HPG) axis are particularly important [1]. The HPA and HPG axes interact with one another in complex way and in part serve to regulate behavior and fertility [2] and [3].

Chiropterans are the second largest order of mammals but the data of measurement of adrenal functions in Chiroptera is reported only from a few species. These results indicate that there are both seasonal and stress related changes in adrenocortical activity [4], [5], [6], [7].

Although the fine structure of adrenal gland is reported in several mammals [8], [9], [10], there is scanty information available on the fine structure of the adrenal gland of bats [11], [12], [13]. Thus the detailed study of the ultrastructural and functional characteristics of the adrenal gland of $T$. longimanus during different phases of the reproductive cycle has been undertaken to find out the probable role of adrenocortio-medullary cells in the control of reproductive physiology of this species of bat.

\section{Materials And Methods}

The emballonurid bat, T. longimanus (Hardwicke) was selected for the present study because of its unique reproductive habits. This bat shows an aseasonal polyestrus (continuous) breeding pattern [14].The specimens were collected from Nagpur, India throughout the year representing different reproductive states. Adult females were trapped alive and were brought alive in the laboratory with minimum stress and constant supply of food and glucose water. Mature females were separated from immature females after observing mammary glands and pelvic dugs. Seven sexually mature females in estrus and five females each in mid- and late pregnancy were killed by cervical dislocation for present investigation.

For the electron-microscopic studies, adrenal glands were immediately removed from non pregnant and pregnant bats after they were killed by decapitation. The tissues were fixed in fresh ice-cold $3 \%$ glutaraldehyde for three hours and then four hours in $0.1 \mathrm{M}$ cacodylate buffer. The tissues were washed in buffer and then post fixed for one to two hours in $1 \% 0.067 \mathrm{M}$ cacodylate-buffered osmium tetroxide. After dehydration with graded series of alcohol, the tissues were cleared in propylene oxide solution and embedded in Araldite resin which 
would be polymerized at $60^{\circ} \mathrm{C}$. Then, ultrathin sections from selected blocks were cut with glass knife and picked up on 400-mesh copper grids. Sections were double stained with $10 \%$ alcoholic uranyl acetate for 20 min and for $10 \mathrm{~min}$ in Reynold's lead citrate. The sections were examined under a JEM Jeol-100s electron microscope (Japan) at $80 \mathrm{KU}$ accelerating voltage and photographed.

\section{Results}

The fine structure of the adrenocortical cells is described, with more emphasis on the organelles involved in the process of steroidogenesis. Adrenal gland of pregnant female shows striking differences in ultrastructural features than those observed in the adrenal gland of non-pregnant female.

During estrus, the zona glomerulosa is made up of a group of four to six cells arranged in circular cords or acini like structures (Fig.1). Each cell is bounded by a plasma membrane of uniform thickness and separated from adjacent cells by a space of constant width. The nuclei of the cells of zona glomerulosa are round to oval shape. The lipid droplets are few in number and usually appear as large vacuoles. These droplets are variable in size, mainly round to ovoid in shape with an irregular boundary containing material of variable electron density. The Golgi complex is inconspicuous. Mitochondria are many, round in shape with vesicular cristae. The smooth endoplasmic reticulum is in the form of small vesicles scattered throughout the cytoplasm. The zona fasciculata constitutes the major portion of the adrenal cortex. These cells are large and arranged in radial cords, one or two cells thick and lie between vascular channels which converge towards the medulla. Mitochondria are pleomorphic showing vesicular cristae. In some, mitochondrial cristae are collapsed. The cells of zona fasciculata contain more number of lipid droplets than those observed in the cells of glomerulosa. These droplets are pleomorphic with irregular boundaries. The cells of zona fasciculata have tubular profiles of smooth endoplasmic reticulum in the form of vesicles scattered throughout the cytoplasm (Fig.2). In contrast to the zona glomeruolosa the cells of the zona fasciculata are rich in smooth endoplasmic reticulum. Free ribosomes are encountered in the cytoplasm. The cells of fasciculata contain large dense bodies (lysosomal bodies). One or more larger granules form a bulge at the periphery of the dense body. Such dense bodies are not observed in the cells of glomerulosa.

There are no marked differences in the ultrastructural characteristics of the zona glomerulosa and zona fasciculata during mid- and late pregnancy of this bat. The zona glomerulosa consists of short looped cords of cells. The nucleus is spherical and somewhat eccentrically placed. A thin rim of chromatin material observed at the periphery of the nucleus. The nucleolus is large and prominent. Golgi apparatus is indistinct during midpregnancy but it is very well developed during late pregnancy. It is made up of arrays of parallel lamellae. Some of the lamellae of Golgi are dilated. A large number of small vesicles, some of which have appearance of coated vesicles are associated with the lamellae of the Golgi apparatus. Mitochondria are large in number and are scattered throughout the cytoplasm. Mitochondria have lamellar cristae in mid-pregnancy but lamellar as well as vesicular cristae in late pregnancy. Tubular profiles of smooth endoplasmic reticulum are seen scattered in the cytoplasm. Lipid droplets show wide variations in size and electron density (Figs. 3, 4).

The cells of the zona fasciculata are large, vacuolated and arranged in groups or cords of polygonal cells. Mitochondria are randomly distributed in the cytoplasm. Number of mitochondria is more than those observed in the glomerulosa. Mitochondria are larger and more pleomorphic than those of the glomerulosa. Mitochondrial cristae are mostly tubular but lamellar form of cristae is also observed. The rough endoplasmic reticulum is rare but large number of free ribosomes is seen. Most striking features of the cytology of this zone are the elaborate development of the smooth endoplasm reticulum and more number of lipid droplets than those observed in the zona glomerulosa. Golgi apparatus is inconspicuous. The lipid droplets are observed in close association with smooth endoplasmic reticulum and mitochondria (Figs. 5, 6).

During estrus, medulla consists of chromaffin cells arranged in small groups or short cords surrounded by blood capillaries and connective tissue. The chromaffin cells which are irregular in outline has eccentrically placed nucleus with peripheral chromatin clumps. Golgi zone is well developed. The rough endoplasmic reticulum is in the form of short and elongated cisternae arranged in parallel profiles. Mitochondria are round or oval and are scattered throughout the cytoplasm. The distinctive features of medullary cells are the chromaffin granules which appear as a membrane bound body of variable electron density. Some cells contain predominantly dense black granules called epinephrine cells, while others show scattered empty vesicles with small amounts of granular material lining to the inner layer or present in the lumen of the vesicles called norepinephrine cells. Thus, both the cell types are observed in medulla of estrus bat as in other mammals (Fig. 7).

During pregnancy, medullary chromaffin cells show similar ultrastructural features exhibited by the medullary cells during estrus. Two types of cells are identical during pregnancy as observed during estrus. However, nor-epinephrine cells are predominant during pregnancy (Fig. 8). 


\section{Discussion}

The adrenal cortex of bats as in mammals is differentiated into three distinct zones: zona glomerulosa, zona fasciculata and zona reticularis. However, the zona reticularis is absent in the adrenal gland of $T$. longimanus but it is present in the form of islets of cortical cells in the medullary region. Similar observations are reported in T. longimanus [15].

The ultrastructural characteristics of the cells of zona glomerulosa of bat adrenal gland show some striking differences during estrus and pregnancy. Golgi apparatus is inconspicuous during estrus but well developed during late pregnancy. Mitochondria show vesicular cristae during estrus and vesicular and lamellar cristae during pregnancy. Moderate to high amounts of tubular profiles of smooth endoplasmic reticulum is seen during estrus. However, there is a extensive development of tubular profiles of smooth endoplasmic reticulum during pregnancy. A few lipid droplets are observed in the cytoplasm during estrus but large numbers of lipid droplets of various size and electron density are seen in the cytoplasm during pregnancy. Similar observations are reported in the glomerulosa cells of bat, $H$. lankadiva during estrus and pregnancy [13] and during hibernation and arousing of dormouse, Muscardinus avellanarius [8] supporting present observations.

The ultrastructure of the zona fasciculata of non-pregnant adult female and pregnant female of bat, $T$. longimanus shows striking differences in adreno-cortical region. The zona fasciculata is more developed in the pregnant animal than the non-pregnant adult female. The zona fasciculata is well developed as compared to other zones of adrenal cortex during both the phases of the cycle. The cells of zona fasciculata of non-pregnant bat show presence of vesicular smooth endoplasmic reticulum, numerous spherical mitochondria with vesicular cristae and lipid droplets indicating that the cells are synthesizing steroid hormones. Zona fasciculata of pregnant bat shows presence of well developed Golgi network, elaborate network of vesicular or tubular cisternae of smooth endoplasmic reticulum, mitochondria with vesicular cristae, and numerous lipid droplets. There is an increase in Golgi-SER area during pregnancy as compared to non-pregnancy. These ultrastructural features indicate that there is an increase in rate of steroid synthesis in the cells of zona fasciculata during pregnancy. The present study corroborates the same finding in bat, $H$. lankadiva during estrus and pregnancy [13].

Adrenocortical cells of $T$. longimanus possessed ultrastructural organelles such as abundant mitochondria with tubule-lamellar cristae, lipid droplets and smooth surface endoplasmic reticulum suggest that they are steroid hormone secreting cells. Our present report corroborates the finding in other mammals [8], [9], [10] and bat [11], [12], [13], [16].

The zona fasciculata of the cortex of the fruit eating bat, Megaloglossus woermanni has some unusual features [16]. The mitochondrial cristae are hexagonal and the rough endoplasmic reticulum is prominent. Smooth endoplasmic reticulum and mitochondria occupied $25 \%$ and lipid bodies $8 \%$ of cytoplasmic volume. These features of the adrenal cortex of this animal are probably adaptive for steroidogenesis. However, in $T$. longimanus, mitochondrial cristae are lamellar and vesicular. The presence of vesicular and lamellar cristae is considered as unique feature of steroid producing cells [17], [18]. In T. longimanus, abundant smooth endoplasmic reticulum is observed in the adrenal cortex of estrus and pregnant bat. The presence of smooth endoplasmic reticulum is characteristic of actively secreting steroid cells and may well reflect an active synthesis of cortisol. The smooth reticulum contains enzymes involved in the synthesis of cholesterol, the conversion of pregnenolone to progesterone and of deoxycorticosterone [19].

The enzyme $\Delta^{5}-3 \beta$ HDS has been localized in the cells of glomerulosa and fasciculata of $T$. longimanus, a continuous breeder [15]. Contrary to this differential enzyme activity is localized and graded in cortical cells of $T$. melanopogon, a seasonal breeder [20]. The enzyme $\Delta^{5}-3 \beta$ HDS has been demonstrated in adrenal gland of bat, $V$. pipistrellus and suggested that adrenal cortex of bat is capable of synthesizing corticosteroids and also sex steroids [21]. The difference in adrenal gland activity in T. longimanus, a continuous breeder and T. melanopogon, a seasonal breeder, seems to relate to their breeding habits. In the present study ultrastructural features typically associated with steroid producing cells, such as large number of lipid droplets together with mitochondria and agranular endoplasmic reticulum are noted in cortical cells of adrenal gland of $T$. longimanus. This suggest that cortical cells are the major site of steroidogenesis as they contain steroid precursor, lipid containing cholesterol or its esters together with steroidogenic enzymes, supporting the above statement.

In T. longimanus, the medullary cells during estrus and pregnancy are characterized by the presence of well developed Golgi apparatus, rough endoplasmic reticulum, large number of mitochondria and chromaffin granules indicating active synthesis of monoamines during estrus and pregnancy. Medulla of $T$. longimanus during estrus and pregnancy shows two types of chromaffin cells as described in rat medulla [22]. The nor epinephrine cells are predominant in the medulla of pregnant bat while epinephrine cells are predominant in the medulla of estrus bat. Medullary secretion in monkey is predominantly epinephrine in man. The present observations suggest that two cell types are present in bat species similar to that observed in mammals [23], [24], [25], [26]. 


\section{Conclusion}

The primary function of the adrenal gland is to protect the organism against acute and chronic stress. In acute stress catecholamines of the medulla mobilize glucose and fatty acids for energy and prepare the heart, lungs and muscles for action. The plasma levels of adrenocorticotropic hormone (ACTH) and glucocorticoid levels increase in pteropid bats in response to standard stresses such as handling or restraints despites very high levels in plasma [7]. Glucocorticoids protect against over reactions of the body's responses to stress and in more chronic stress of food and fluid deprivation, stimulate glyconeogensis to maintain the supply of glucose [1]. Stress as well as hyper and hypofunction of adrenal gland is known to suppress reproduction in mammals and in women is associated with anovulation and amenorrhea, [27].

In the present study, we did not measure the plasma ACTH and glucocorticoid levels in T. longimanus however, ultrastructural characteristics of ACTH cells, [28], the adrenocortico-medullary cells and enzyme $\Delta^{5}$ $3 \beta \mathrm{HDS}$ in the glomerulosa and fasciculata cells of T. longimanus [15] during estrus and pregnancy suggest that these cells are equipped with the all infrastructure necessary for synthesis of steroid hormones. The HPA and HPG are very active during estrus and pregnancy of this species of bat. The adrenal gland of $T$. longimanus shows seasonal and stress related changes during estrus and pregnancy. Thus the adrenal gland of bat is not only responsible for synthesis of glucocorticoids, sex steroid hormones but also catecholamine that are required to protect bat species against acute and chronic stress as well as to support reproductive processes of this species of bat. We do not have evidence to support either of these possibilities at this time. Further studies are needed to substantiate the hypothesis.

\section{ACKNOWLEDGEMENTS}

The electron microscopy facilities provided by Dr. Arun Chitale, Department of Histopathology, Jaslok Hospital and Research Centre, Mumbai, are gratefully acknowledged. Our thanks are due to Mr. Dilip Kanaskar and Shivaji Bhosale for their excellent technical assistance.

\section{References}

[1] D Reeder and K. Kramer, Stress in free-ranging mammals: Integrating physiology, ecology, and natural history, Journal of Mammalogy, 86, 2005, 225-235.

[2] A DeVries, Interaction among social environment, the hypothalamic-pituitary-adrenal axis and behavior, Horm. Behav, 41, 2002, $405-413$.

[3] V Viau, Functional cross-talk between the hypothalamic-pituitary-gonadal and -adrenal axes, Journal of Neuroendocrinology, 14, 2002, 506-513.

[4] A Gustafson and W. Belt, The adrenal cortex during activity and hibernation in the male little brown bat, Myotis lucifugus lucifugus: annual rhythm of plasma cortisol levels, Journal of Comparative Endocrinology, 44, 1981, 269-278.

[5] E Widmaier and T. Kunz, Basal, diurnal, and stress-induced levels of glucose and glucocorticoids in captive bats, Journal of Experimental Zoology, 265, 1993, 533-540.

[6] E Widmaier, T. Harmer, A. Sulak and T. Kunz, Further characterization of the pituitary-adrenocortical responses to stress in Chiroptera, Journal of Experimental Zoology, 269, 1994, 442-449.

[7] D Reeder, H. Raff, T. Kunz and E. Widmaier, Characterization of pituitary-adrenocortical activity in the Malayan flying fox (Pteropus vampyrus), Journal of Comparative Physiology (B), 176, 2006, 513-519.

[8] C Zancanaro, M. Malatesta, P. Vogel and S. Fakan, Ultrastructure of the adrenal cortex of hibernating, arousing and euthermic dormouse, Muscardinus avellanarius, Anatomical Record, 249, 1997, 359-364.

[9] G Vinson, Adrenocortical zonation and ACTH, Microscopy Research and Technique, 61, 2002, 227-239.

[10] S Tokar, E. Koval, E. Yavorskaand and M. Lukyanetz, Ultrastructural characteristics of lipid droplets in rat adrenocortical cells from zona fasciculata-reticularis, Fiziol Zh, 50, 2004, 107-13.

[11] H Planel, A. Guilhem and J. Soleihavoup, Le cycle annual du cortex surrenal d'un semi-hibernat: Miniopterus schreibersii, Comptes Rendus de I' Association des Anatomistes, 47, 1961, 620-633.

[12] G Romita and R. Montesano, Aspetti isotchimici ed ultrastrutturali delle ghiandole surrenali di Chirotteri durante I'ibernazione, il risveglio ed il periodo estivo, Ateneo Parmense- Sezione i Acta Bio-Medica, 43, 1972 (b), 211-235.

[13] E. Seraphim, Endocrine interaction during different phases of the female reproductive cycle in Hipposideros lankadiva (Kelaart), doctoral diss., RTM Nagpur University, Nagpur (India), 2004.

[14] A Gopalakrishna, Observations on the breeding habits and ovarian cycle in the Indian sheath tailed bat, Taphozous longimanus (Hardwicke), Proc. National Institute of Science, India, 1955, 29-41.

[15] M Lowry, J. Bhardwaj and S. Lall, Enzymorphologic demonstration of $\Delta^{5} 3 \beta$ hydroxysteroid dehydrogenase and succinic dehydrogenase in the cortical and medullary cells of the adrenal gland of Taphozous longimanus Hardwicke (Micorchiroptera: Mammalia), Current Science, 50, 1980, 730.

[16] K Mensah, W. Kesse and A. Ayettey, Ultrastructural features of the zona fasciculata of the adrenal cortex of the fruit eating bat Megaloglossus woermann, West African Journal of Anatomy, 2, 1994, 1-8.

[17] M Hanaki, K. Tanaka and Y. kashima, Scanning electron microscopic study on mitochondrial cristae in the rat adrenal cortex, Journal of Electron Microscopy, 34, 1985, 373-380.

[18] F Prince, Lamellar and tubular associations of the mitochondrial cristae: Unique forms of the cristae present in steroid producing cells, Mitochondrion, 1, 2002, 381-389.

[19] P Nickerson, Formation of concentric whorls of rough endoplasmic reticulum in the adrenal gland of the Mongolian gerbil, Journal of Anatomy, 124 (2), 1977, 383-391.

[20] M Lowry and S. Lall, Morphometric and enzymologic changes in the adrenal cortex and medulla of Taphozous melanopogon melanopogon (Temnick) from nulliparous to the parous state, Myotis, 1986, 23-24.

[21] S Saidapur and V. Nadkarni, Histochemical observations on the adrenal gland of bat Vesperugo pipistrellus (Dobson), Endokrinologie 67, 1976, 244-247. 
[22] G Brown, L. Grota, D. Penney and S. Reichlin, Pituitary adrenal function in the squirrel monkey. Endocrinology, 86, 1970, 519529.

[23] R Coupland, Electron microscopic observations on the structure of the rat adrenal medulla I, The ultrastructure and organization of chromaffin cells in the normal adrenal medulla, Journal of Anatomy, 99, 1965a, 231-254.

[24] R Brenner, Fine structure of adrenocortical cells in adult male rhesus monkeys, American Journal of Anatomy, 119, 1966, 429453.

[25] R Coupland and B.Weakley, Developing chromaffin tissue in the rabbit: an electron microscopic study, Journal of Anatomy, 102, $1968,425-455$

[26] A Belloni, G. Mazzocchi, F. Mantero, and G. Nussdorfer, The human adrenal cortex: ultrastructure and base-line morphometric data, Journal of Submicroscopy and Cytology, 19, 1987, 657-668.

[27] P Green, C. Wilkinson and S. Woods, Intraventricular corticosterone increases the rate of body weight gain in underweight adrenalectomized rats, Endocrinology, 130, 1992, 269-275.

[28] A Nerkar, and M. Gadegone, Ultrastructure of the pars distalis of the Indian female sheath-tailed Bat, Taphozous longimanus (Hardwicke), International Journal of Morphology, 28, 2010, 787-801.

\section{EXPLANATION OF FIGURES}

Figure 1. Electron micrograph showing group of cells of zona glomerulosa during estrus. Note round to oval shaped nuclei (N) and several lipid droplets (LD). X 6000

Figure 2. Electron micrograph of the cell of zona fasciculata during estrus. Note some lysosomal bodies (Ly), several round shaped lipid droplets (LD) associated with several round to elongated mitochondria (M) with vesicular cristae. Some mitochondria are hypertrophied with collapsed cristae and tubular profiles of smooth endoplasmic reticulum (SER) are scattered throughout the cytoplasm. X 12000

Figure 3. Electron micrograph of the cell of glomerulosa during mid-pregnancy showing several round to elongated mitochondria (M) with vesicular cristae. Few lipid droplets (LD) and vesicular profiles of smooth endoplasmic reticulum are seen in the cytoplasm. X 8000

Figure 4. Electron micrograph of the cell of zona glomerulosa during late pregnancy showing few spherical lipid droplets (LD) and several round to oval shaped mitochondria (M) with vesicular cristae. A well developed Golgi apparatus (G) and network of cisternae of smooth endoplasmic reticulum are seen within cytoplasm. X 10000

Figure 5. Electron micrograph of the cell of the zona fasciculata during mid- pregnancy showing several spherical to oval shaped mitochondria (M) with lamellar and tubular cristae. Dense bodies (DB) and lipid droplets (LD) are present in the cytoplasm. Note well developed smooth endoplasmic reticulum (SER) is in the form of vesicles scattered throughout the cytoplasm. X 12000

Figure 6. Electron micrograph of cell of zona fasciculata during late pregnancy showing several spherical lipid droplets (LD) which are observed in close association with smooth endoplasmic reticulum (SER) and mitochondria (M) with tubular cristae. X 10000

Figure 7. Electron micrograph of medullary cell during estrus showing Epinephrine (EP) granules, well developed Golgi apparatus $(\mathrm{G})$ and large vacuolated mitochondria $(\mathrm{M})$ with collapsed cristae. X 12000

Figure 8. Electron micrograph of medullary cell during pregnancy. Note Golgi apparatus (G), parallel cisternae of rough endoplasmic reticulum (RER), large hypertrophied mitochondria (M) with collapsed cristae and norepinephrine (NEP) granules in the cytoplasm. X 12000 